\title{
Feasibility of a polydioxanone plate as an adjuvant material in rhinoplasty in Asians
}

\author{
Gwang Jin $\mathrm{Oh}^{1}$, Jaeik Choi ${ }^{2}$, Taek-Kyun Kim³ , Jae-Yong Jeong ${ }^{3}$, Joo-Hak Kim², Sunje Kim², \\ Sang-Ha $\mathrm{Oh}^{2,4}$ \\ ${ }^{1}$ Namu Plastic Surgery, Seoul; ${ }^{2}$ Department of Plastic and Reconstructive Surgery, Chungnam National University School of Medicine, \\ Daejeon; ${ }^{3}$ The PLUS Plastic Surgery, Seoul; ${ }^{4}$ Brain Research Institute, Chungnam National University College of Medicine, Daejeon, Korea
}

Background Nasal framework-supporting procedures such as septal extension grafts, derotation grafts, and columellar strut grafts are usually required in rhinoplasty in Asian patients because the skin envelope is tight, but the nasal framework is small and weak. Autologous materials are preferred, but they have some limitations related to the amount that can be harvested and the frequency of use. Therefore, synthetic materials have been used to overcome these limitations.

Methods A total of 114 patients who received a polydioxanone (PDS) plate as an adjuvant material in rhinoplasty from September 2016 to August 2017 were retrospectively investigated. The PDS plate was used as to support the weak framework and to correct the contour of the alar cartilages. The PDS plate was used for reinforcement of columellar struts and septal L-struts, alar cartilage push-down grafts, fixation of septal extension grafts, and correction of alar contour deformities

Results Primary and secondary rhinoplasty was performed in 103 and 11 patients, respectively. Clinically, no significant inflammation occurred, but decreased projection of the tip was observed in seven patients and relapse of a short nose was noted in five patients.

Conclusions PDS plates have been used in the United States and Europe for more than 10 years to provide a scaffold for the nasal framework. These plates can provide reinforcement to columellar struts, L-struts, and septal extension grafts. In addition, they can assist in deformity correction. Therefore, PDS plates can be considered a good adjuvant material for Asian patients with weak and small nasal cartilage.

Keywords Asian / Rhinoplasty / Polydioxanone

\author{
Correspondences: Sunje Kim \\ Department of Plastic and \\ Reconstructive Surgery, Chungnam \\ National University School of \\ Medicine, 282 Munhwa-ro, Jung-gu, \\ Daejeon 35015, Korea \\ Tel: +82-42-280-7381 \\ Fax: +82-42-280-7384 \\ E-mail: kkk9243@naver.com \\ Sang-Ha Oh \\ Department of Plastic and \\ Reconstructive Surgery, Chungnam \\ National University School of \\ Medicine, 282 Munhwa-ro, Jung-gu, \\ Daejeon 35015, Korea \\ Tel: +82-42-280-7387 \\ Fax: +82-42-280-7384 \\ E-mail:djplastic@cnu.ac.kr
}

\begin{abstract}
This work was supported by the research fund of Chungnam National University and was supported by the Basic Science Research Program through the National Research Foundation of Korea (NRF) funded by the Ministry of Science, ICT, \& Future Planning (NRF-2018R1A2B6007425).
\end{abstract}

\section{INTRODUCTION}

In Asians, the skin and soft tissue envelope of the nose are strong and tight, but the cartilage is scant and weak. Therefore, a bulbous tip, small nose, and short nose are frequently seen, re- quiring framework support $[1,2]$. Autologous, homologous, and synthetic materials are used for framework support. Among these materials, autologous tissue is preferred, but its harvest faces limitations, such as the amount and quality of cartilage, as well as structural faults at the donor site. Synthetic materials 
have therefore been used to overcome these limitations.

Adjuvant materials recently used in rhinoplasty include porous high-density polyethylene sheets (Medpor), polycaprolactone (PCL), polylactic-co-glycolic acid (PLGA), poly-L-lactic acid (PLLA), and polydioxanone (PDS) plates. In this study, a PDS plate was used as an adjuvant material for the cartilage framework during rhinoplasty. The PDS plate was made of the same material as PDS threads and was approved by regulatory authorities in Europe in 2009, the United States in 2010, and Korea in $2016[3,4]$.

A PDS plate remains structurally intact until 10 weeks after implantation and serves as a temporary scaffold. After 25 weeks, the plate is completely degraded without significant effects on the surrounding structures $[5,6]$. A PDS plate also guides cartilage regeneration, so that new chondrocytes are arranged regularly at the defect area, which prevents cartilage deviation [4]. Using a PDS plate together with cartilage results in increased strength of the cartilage and PDS plate complex. For example, using a $0.15-\mathrm{mm}$ PDS plate with cartilage was found to yield a 3- to 4-fold increase in strength compared to the cartilage alone $[3,7]$.

\section{METHODS}

This study was approved by the Research Ethics Board at Chungnam National University Hospital (IRB No. 2018-08-066) and informed consent was obtained.

\section{Patients}

A PDS plate was used in 114 patients, consisting of 100 women (87.7\%) and 14 men (12.3\%), over a 1 -year period extending from September 2016 to August 2017. The patients' age ranged from 17 to 57 years (mean age, $30.8 \pm 8.4$ years). PDS plates were used to reinforce the columellar strut or the remaining Lstrut after septoplasty, fixation of a septal extension graft, and correction of alar cartilage (Table 1).

\section{Surgical techniques}

\section{Reinforcement of the columellar strut}

If any deviation or weakness was observed on the harvested ear or nasal septum when making a columellar strut, it was reinforced with a PDS plate. The PDS plate was cut to the length of the required columellar strut; the size was $10 \times 20 \mathrm{~mm}$ in most cases. The three sides of the columellar strut were wrapped with the PDS in a U-shape. The PDS plate was fixed to the columellar strut using a PDS suture $(5 / 0$ or 6/0) (Fig. 1).

\section{Reinforcement of the remaining L-strut after septoplasty}

The main concern after septoplasty is whether the remaining L-

Table 1. Demographic data of the 114 patients in the study

\begin{tabular}{|lc|}
\hline Characteristic & Value \\
\hline Sex & \\
Female & $100(87.7)$ \\
Male & $14(12.3)$ \\
Age (yr) & $30.8 \pm 8.4$ \\
Previous treatment & \\
Primary rhinoplasty & $103(90.4)$ \\
Secondary rhinoplasty & $11(9.6)$ \\
Donor site & \\
Ear & $104(91.2)$ \\
Nasal septum & $10(8.8)$ \\
\hline Values are presented as number (\%) or mean \pm SD. & \\
\hline
\end{tabular}

\section{Fig. 1. Reinforcement and straightening of the columellar strut}

(A) Slight deviation shown on the columellar strut in the inferior portion created with bilayered ear cartilage. The columellar strut was wrapped with polydioxanone (PDS) in a U-shape and fixed to the columellar strut using a PDS suture (5/0). (B) The corrected columellar strut could be reinforced and straightened using the U-shaped PDS plate.
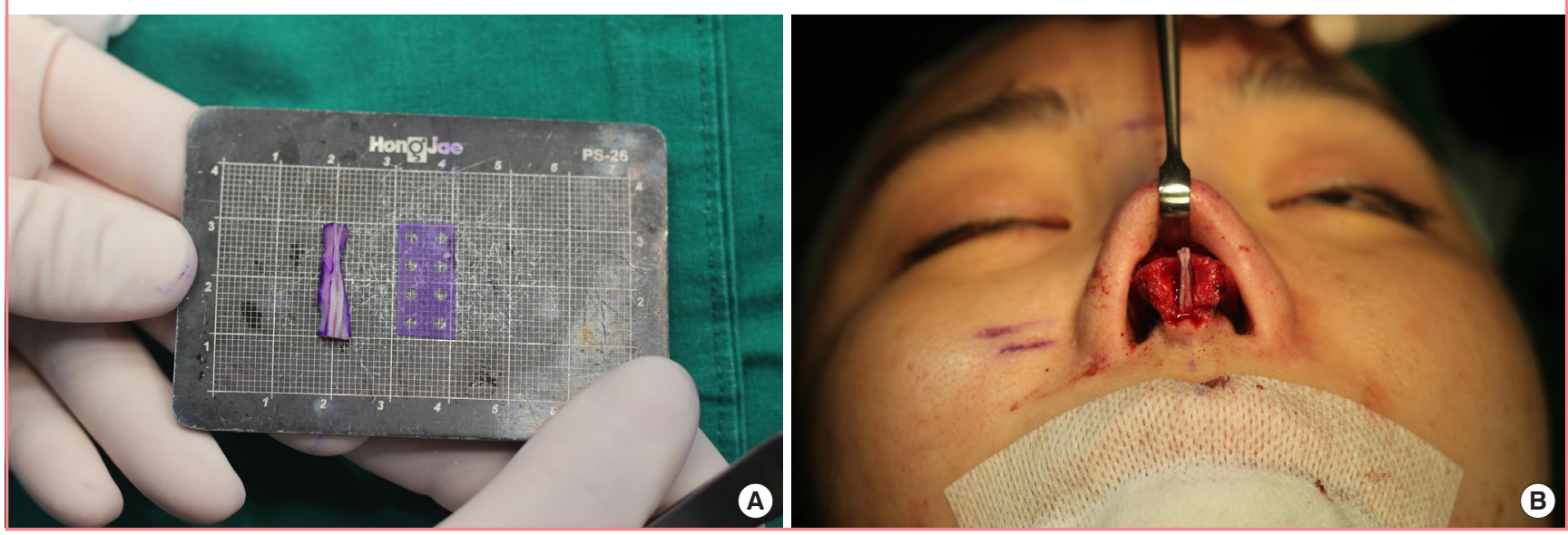
strut is strongly maintained. The nasal septum of Asians is usually small or thin, so more attention is required. After septoplasty, a PDS plate can be used to reinforce the remaining L-strut and to reduce the risk of L-strut collapse or weakening. In this study, the PDS plate was cut to form an L-shape and attached to one side of the remaining L-strut (Fig. 2).

\section{Alar cartilage push-down graft}

When the alar cartilage was barely advanced or bent with a tip extension suture due to insufficient strength or size of the cartilage, a PDS plate was used to support the tip extension suture and to push down the alar cartilage. The PDS plate was cut into a tripod shape and fixed to the upper lateral and alar cartilage (Fig. 3). In addition, it can be used along with a de-rotation graft when additional support is required.

\section{Support and fixation of the septal extension graft}

A PDS plate was used to support and fix the septal extension graft. The PDS plate was attached to both or one side of the septal extension graft, as a batten or spreader type. This allowed stable end-to-end placement of septal extension grafts and the Lstrut. Minor septal deviations could be also corrected by scoring the concave side of the septum and then attaching the PDS plate to the septum for reinforcement (Fig. 4).

\section{Fig. 2. Reinforcement of the remaining L-strut after septoplasty}

A polydioxanone (PDS) plate was cut into a L-shape with a size of $25 \times 15 \mathrm{~mm}$. The PDS plate was attached to one side of the remaining L-strut.

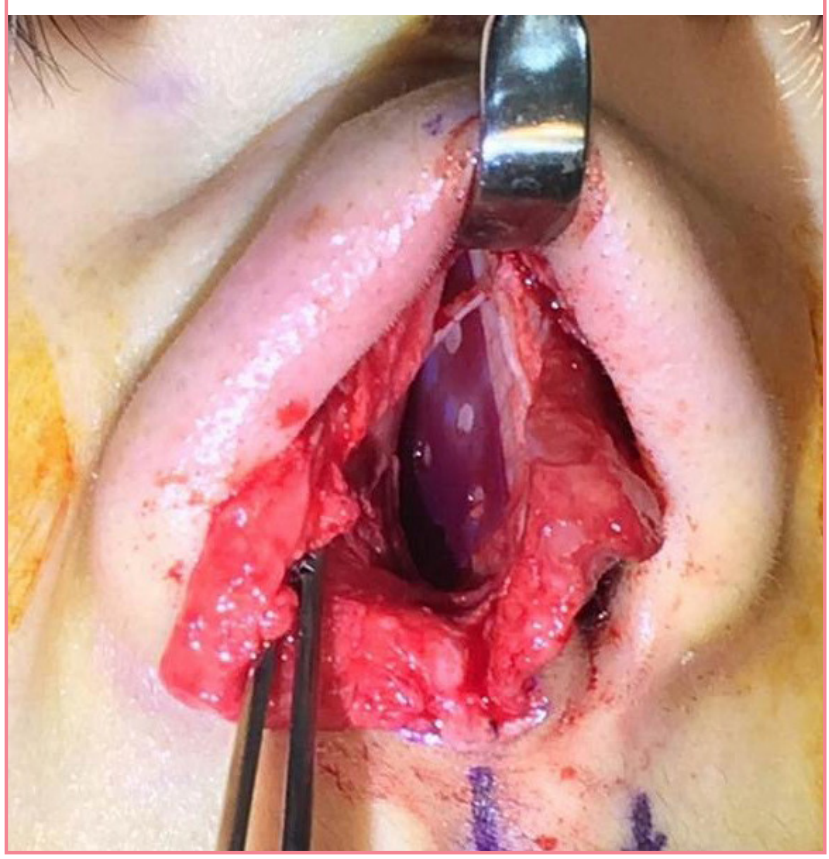

\section{Alar cartilage contour correction}

A PDS plate can be used to correct defects in the cartilage shape, such as dome buckling and height discrepancy of the alar carti-

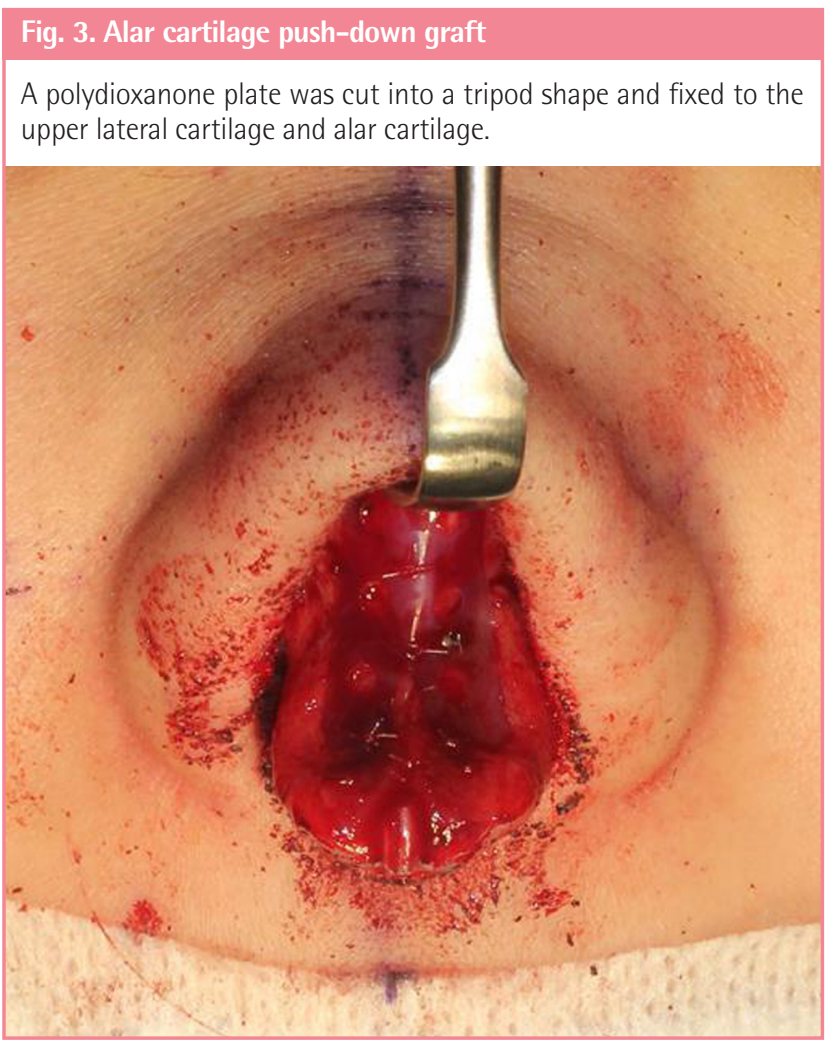

Fig. 4. Support and fixation of a septal extension graft

A polydioxanone plate was attached to both or one side of the septal extension graft, as a batten or spreader type.

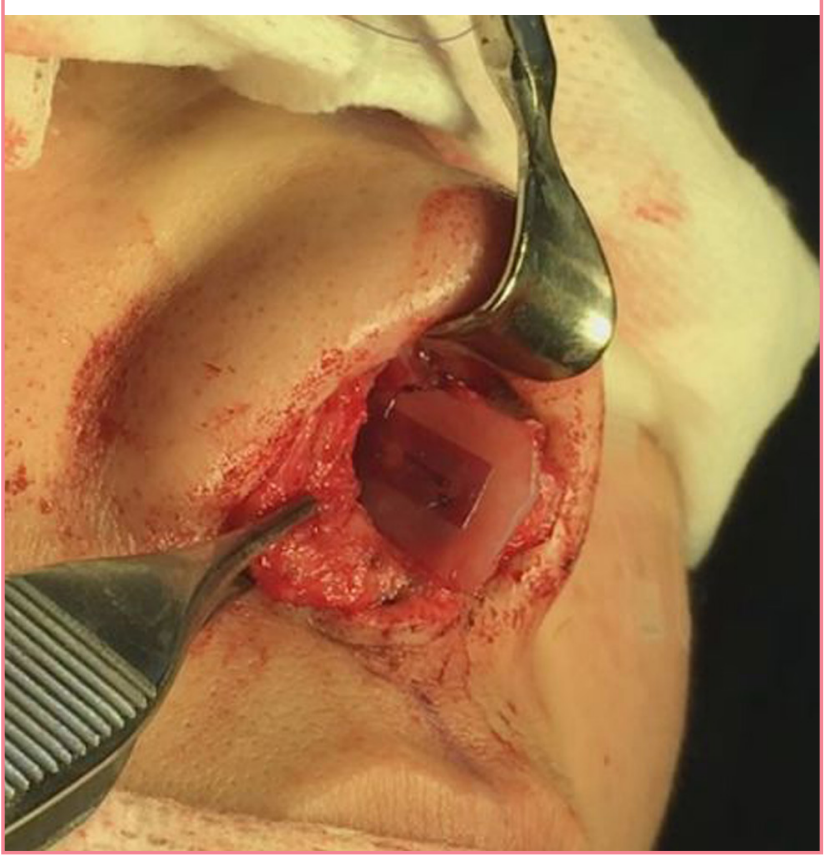


Fig. 5. Alar cartilage contour correction

(A) An asymmetric and curved deformity was seen in the left alar cartilage. (B) After correction using the suture method, a polydioxanone plate was attached to the deformed area.
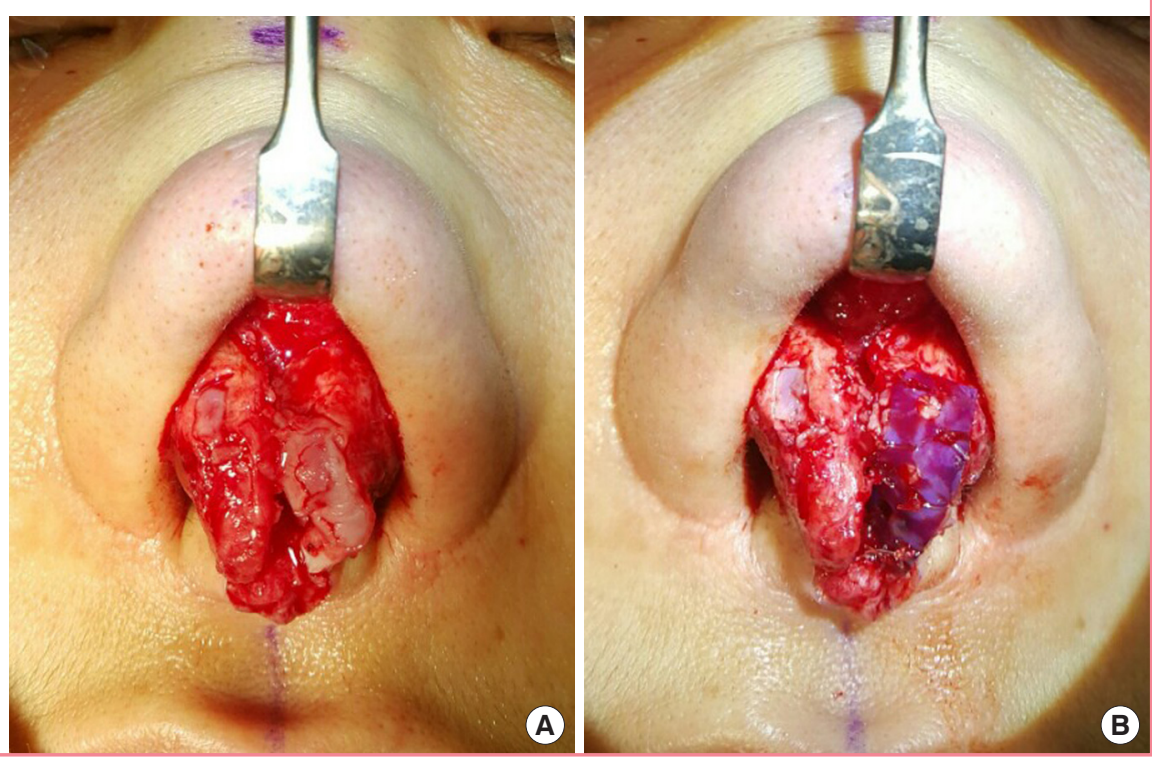

\section{Fig. 6. Alar cartilage contour correction: umbrella shape}

When a height discrepancy between the domes or pinched tip deformity was found, the columellar strut was reinforced using a polydioxanone plate with an umbrella shape.

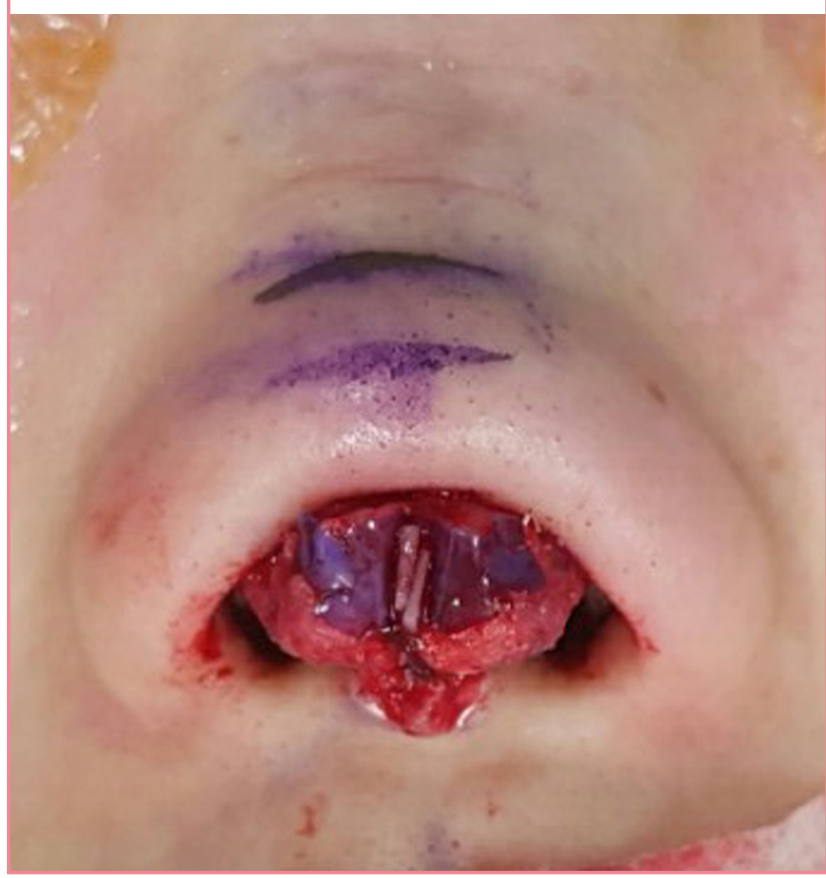

lage, to provide additional support, or to alleviate irregularities after correction. In this series, after the curved cartilage was corrected as much as possible using the suture method, a PDS plate was attached to the deformed area (Fig 5). When a height discrepancy between the domes or a pinched tip deformity was found, the columellar strut was reinforced using a PDS plate with an umbrella shape (Fig. 6).
Table 2. Applications of the polydioxanone plate in the 114 patients

\begin{tabular}{|lc|}
\hline Application & No. of patients $(\%)^{\text {a) }}$ \\
\hline Reinforcement of the columellar strut & $106(92.9)$ \\
Reinforcement of the remaining L-strut after septoplasty & $9(7.9)$ \\
Alar cartilage push-down graft & $22(19.2)$ \\
Supporting and fixing of a septal extension graft & $14(12.2)$ \\
Alar cartilage contour correction & $5(4.4)$ \\
\hline a)The total number of patients is different from the sum of the above numbers \\
because there were cases where two or more of the above procedures were \\
performed in the same patient.
\end{tabular}

Table 3. Complications in the 114 patients

\begin{tabular}{|lc|}
\hline Complication & No. of patients (\%) \\
\hline Infection & 0 \\
Extrusion & 0 \\
Septal perforation & 0 \\
Local swelling and erythema & $3(2.6)$ \\
Decreased projection of the nasal tip & $7(6.1)$ \\
Short nose deformity & $5(4.4)$ \\
\hline
\end{tabular}

\section{RESULTS}

Primary rhinoplasty was performed in 103 patients and secondary rhinoplasty was conducted in 11 patients. The donor sites of the cartilage were the ear $(n=104)$ and nasal septum $(n=10)$. PDS plates were used to reinforce the columellar strut in 106 cases and to reinforce the remaining L-strut after septal surgery in nine cases. The PDS plates were also used for creation of an alar cartilage push-down graft in 22 cases, for support and fixation of a septal extension graft in 14 cases, and for alar cartilage 
Fig. 7. Reinforcement of the remaining L-strut

(A) A 22-year-old woman with weak alar cartilage underwent augmentation with a silicone implant and tip-plasty including an onlay graft and columellar strut graft with nasal septal cartilage. A polydioxanone plate was used to reinforce the columellar strut and remaining L-strut septum. (B) Four-month postoperative view.
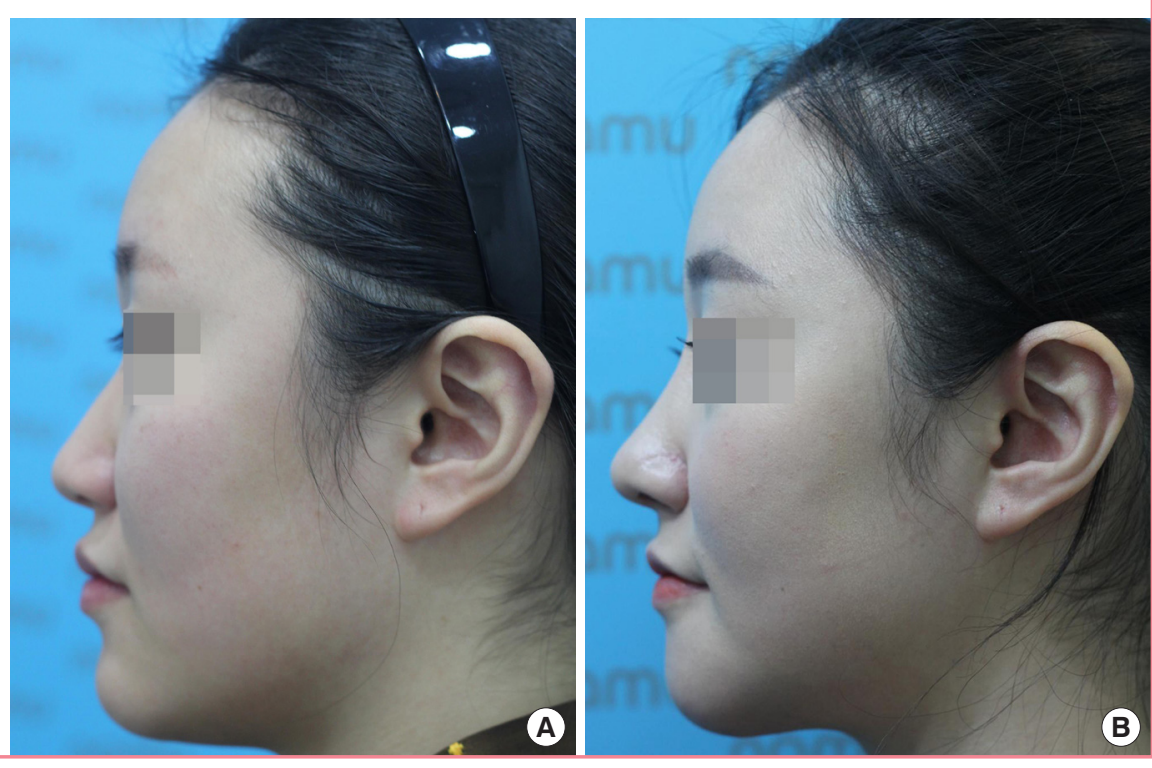

Fig. 8. Alar cartilage push-down graft

(A) A 24-year-old woman with a short nose underwent secondary rhinoplasty with implant exchange, tip-plasty, and a lower lateral cartilage push-down graft. A polydioxanone plate was used to reinforce the columellar strut and the lower lateral cartilage pushdown graft. (B) Six-month postoperative view.
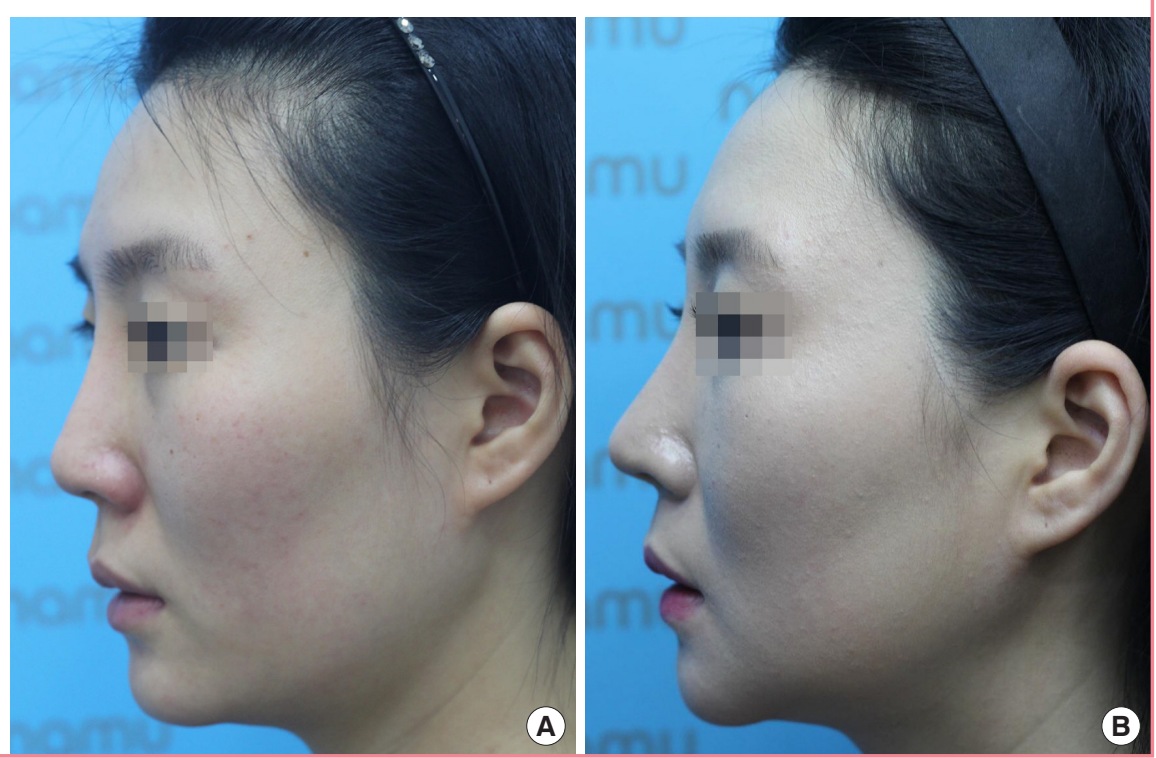

correction in five cases (Table 2). No evidence of postoperative infection or inflammation was observed (Figs. 7-9). Three patients had erythema and local swelling around the columellae, which improved with conservative treatment. Decreased projection of the nasal tip was noted in seven patients. In addition, relapse of a short nose occurred in five patients (Table 3 ).

\section{DISCUSSION}

Because of the tight skin and weak cartilage structure of Asians, framework changes and support are important components of most rhinoplasty procedures in Asian patients. Various materials can be used for correction, of which autologous tissues are preferred in rhinoplasty because they are highly biocompatible and have a low risk of infection or extrusion compared to homologous or alloplastic materials [8].

The most common material used to support the framework is the nasal septum. The nasal septum is composed of hyaline cartilage with similar properties to the native cartilaginous framework of the nose itself [8]. Because the nasal septum is located in the same operative field, an additional incision is unnecessary, which reduces the operating time. In addition, the nasal septum is straight and strong, so it can endure the tensile force of the skin and soft tissue envelope. However, if the septum is small 


\section{Fig. 9. Alar cartilage contour correction}
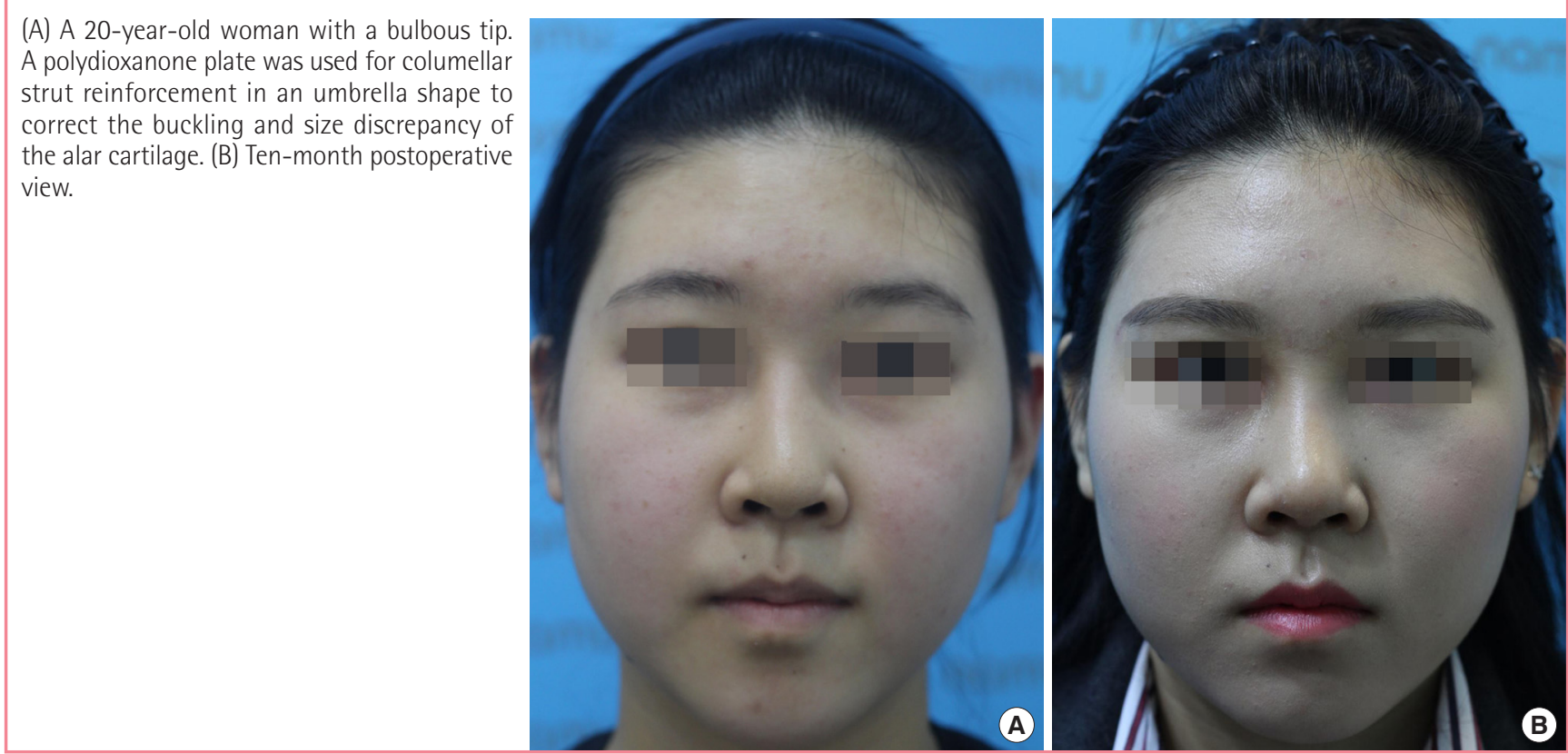

and thin, the harvesting procedure itself is difficult and the amount harvested might be insufficient. This might lead to subsequent collapse or weakening of the L-strut. For the same reason, harvesting is not possible if the nasal septum has already been harvested in a previous operation. Another autologous material used for framework support is the ear cartilage. The ear cartilage is an elastic cartilage that is used mainly as an onlay graft, rather than as framework support, due to its innate curved shape. Nevertheless, an incision is required on the auricle because the donor site is not in the same operative field. This could cause hypertrophic scarring or a deformity at the donor site. Rib cartilage can provide both a sufficient amount of cartilage and strong support $[9,10]$. However, harvesting rib cartilage leaves a scar on the chest wall and extends the operating time. In addition, the pleura could be perforated during harvest.

Various synthetic materials have been developed and used to overcome the limitations of autologous tissues. The ideal synthetic materials should be non-inflammatory, non-carcinogenic, non-allergenic, resistant to mechanical strain, able to be integrated into the host tissue, readily available, easy to shape, and fully sterilizable. No synthetic material satisfies all of these properties. Representative examples of synthetic materials include Medpor, PCL, PLGA, PLLA, and PDS plates. Medpor has many advantages, such as being thin, strong and easy to manipulate [11]. However, it might damage and adhere to the surrounding tissues; thus, it is difficult to remove during a subsequent operation. Medpor is also non-absorbable [12]. Polymers, such as PCL, PLGA, and PLLA, are biodegradable, but the absorption period can be as long as 1 to 2 years. Resected cartilage appears to regenerate after 10 weeks. Implants are necessary only as long as the supporting tissue heals; afterward, they need to be removed to avoid long-term complications. In addition, they are thicker than Medpor, potentially damaging the surrounding tissues or causing inflammation during the long absorption period $[4,6,7]$.

PDS is a colorless, crystalline, and biodegradable polymer that is used mainly as a suture material and can be made in the form of a sheet [13]. PDS plates are available in three types defined in terms of thickness and perforation: $0.15 \mathrm{~mm}$ (perforated), 0.25 $\mathrm{mm}$ (perforated), and $0.5 \mathrm{~mm}$ (unperforated). These plates remain structurally intact for up to 10 weeks after implantation and serve as a scaffold. After 25 weeks, they are degraded completely by hydrolysis and metabolized with no damage to the surrounding tissues. Moreover, they do not cause any inflammatory or foreign body reactions $[4,5]$. Another important feature of PDS plates is their ability to guide cartilage regeneration [7]. This plate is flexible and light, and has the mechanical properties of retaining memory and stiffness when an external force is applied. Therefore, it has been used to stabilize the nasal cartilage framework structures $[3,5,14]$.

A 0.15-mm perforated PDS plate was applied as an adjunct to reinforce the columellar struts or L-struts and alar cartilage push-down grafts, as well as for septal extension graft fixation and alar contour correction. The 0.15 -mm perforated PDS plate retains its stiffness for 10 weeks after implantation, which is a sufficient interval for the grafted cartilage to be remodeled and 


\section{Fig. 10. Comparison of the PDS plate and Suture method}

A suture (red X) has only one point of contact, but the plate (red rectangle) has a large area of contact, making it more robust for shape correction and maintenance. PDS, polydioxanone.
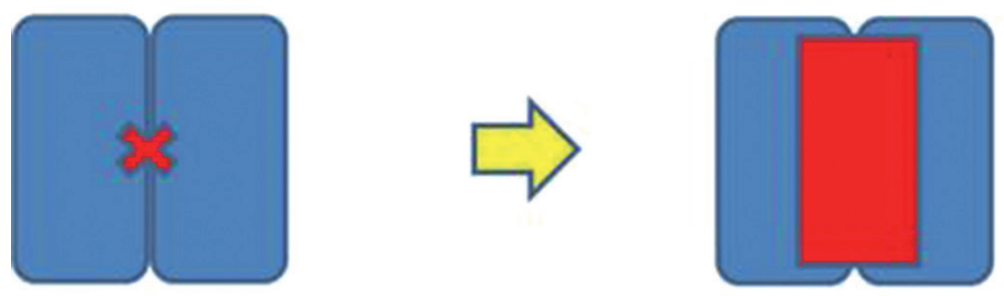

or

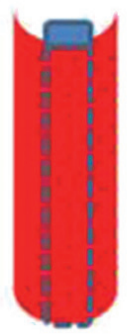

enveloped by connective tissue [3,4]. In addition, blood and nutrients can be supplied to the cartilage through the perforations, providing a favorable environment for the survival of the grafted cartilage [15]. If a columellar strut made of ear cartilage or the nasal septum is weak or bent, a PDS plate can be wrapped around the strut in the form of a U-shape. Definite improvements in strength and shape could be confirmed under direct vision and palpation (Fig. 7).

The septal cartilage of the nose is the most common material used in dorsal lengthening procedures to correct a short nose. However, the cartilaginous framework of Asians is often weak and thin; thus, the amount of harvestable septum is insufficient in most patients with a short nose. Therefore, excessive harvesting can occasionally induce a saddle nose. In addition, although the L-strut is sufficient, correction should be performed using a scoring procedure when deviation occurs. For these reasons, the stability of an L-strut cannot be guaranteed. To prevent L-strut weakness, the PDS plate is attached to the remaining L-strut to enhance the strength of the remnant L-strut and to reduce the possibility of collapse. Moreover, the corrected nasal septum can be maintained in a straight form after reduction because the PDS plate acts as a splint (Fig. 8).

A tip extension suture is another useful tool for Asian patients [16]. When a PDS plate is used in tip correction, the contact area is the surface, so that the retention force is superior to the suturing method alone. In addition, the PDS plate can act as a scaffold or splint for weak or deformed alar cartilage, enabling it to maintain a favorable shape and strength (Fig. 9).

The septal extension graft can be fixed as a spreader or batten type. In this manner, a PDS plate is placed over the graft to facilitate fixation and to increase the strength of the graft. The PDS plate also allows end-to-end placement of a septal extension graft, which reduces the extent of septal bulging of a bilateral spreader or batten graft. The PDS plate retains similar memory and stiffness to the ear cartilage, so it can be used to correct the alar contour. In addition, it can be used for deformities of the upper lateral cartilage, such as inner valve collapse.

Compared to the line or point force exerted by a suture, the membrane-type PDS plate is advantageous because it has a larger contact surface (Fig. 10).

As a recommendation based on our experiences, the PDS plate should be have a rounded-off edge to prevent palpation and exposure, particularly at the nasal tip and anterior columella, because the plate can be palpable or exposed if its cutting edge is sharp. Additionally, the authors recommend that an objective evaluation should be conducted over a relatively long follow-up period (more than 1 year) with accurate measurements of the tip projection and columello-labial angle.

The PDS plate has been used in Europe and the United States for more than a decade, and it is a safe material in plastic surgery. It provides a scaffold for autologous tissue not as a point, but as a surface, resulting in greater strength. In addition, as a frame support, it can also be used for a range of applications, such as contour correction. Therefore, it is a good alternative synthetic material, particularly for Asian patients with weak and deficient nasal cartilage.

\section{NOTES}

\section{Conflict of interest}

No potential conflict of interest relevant to this article was reported.

\section{Ethical approval}

The study was approved by the Institutional Review Board of Chungnam National University Hospital (IRB No. 2018-08066) and performed in accordance with the principles of the Declaration of Helsinki. Written informed consents were obtained. 


\section{Patient consent}

The patients provided written informed consent for the publication and the use of their images.

\section{Author contribution}

Study concept and design: Oh GJ. Data acquisition: Kim TK, Jeong JY. Data analysis and interpretation: Choi J. Drafting of the manuscript: Oh GJ, Kim S. Critical revision of the manuscript for important intellectual content: Kim S, Oh SH. Statistical analysis: Kim JH. Study supervision: Kim S, Oh SH. Approval of final manuscript: all authors.

\section{ORCID}

Gwang Jin Oh https://orcid.org/0000-0001-6133-562X Jaeik Choi https://orcid.org/0000-0002-4813-5428 Taek-Kyun Kim https://orcid.org/0000-0002-3213-9803 Jae-Yong Jeong https://orcid.org/0000-0001-5654-4443 Joo-Hak Kim https://orcid.org/0000-0001-9244-0940 Sunje Kim https://orcid.org/0000-0001-7887-7521 Sang-Ha Oh https://orcid.org/0000-0003-3734-5005

\section{REFERENCES}

1. Matory WE Jr, Falces E. Non-Caucasian rhinoplasty: a 16year experience. Plast Reconstr Surg 1986;77:239-52.

2. Kim DA, Jeong JY, Oh SH. Bulbous tip correction focusing on skin soft tissue envelope in Asian rhinoplasty. Arch Aesthetic Plast Surg 2014;20:140-7.

3. Conderman C, Kinzinger M, Manuel C, et al. Mechanical analysis of cartilage graft reinforced with PDS plate. Laryngoscope 2013;123:339-43.

4. Boenisch M, Mink A. Clinical and histological results of septoplasty with a resorbable implant. Arch Otolaryngol Head Neck Surg 2000;126:1373-7.

5. Boenisch M, Tamas H, Nolst Trenite GJ. Influence of polydi- oxanone foil on growing septal cartilage after surgery in an animal model: new aspects of cartilage healing and regeneration (preliminary results). Arch Facial Plast Surg 2003;5: 316-9.

6. Rimmer J, Ferguson LM, Saleh HA. Versatile applications of the polydioxanone plate in rhinoplasty and septal surgery. Arch Facial Plast Surg 2012;14:323-30.

7. Kim JH, Wong B. Analysis of cartilage-polydioxanone foil composite grafts. Facial Plast Surg 2013;29:502-5.

8. Sajjadian A, Rubinstein R, Naghshineh N. Current status of grafts and implants in rhinoplasty: part I. autologous grafts. Plast Reconstr Surg 2010;125:40e-49e.

9. Kim JH, Song JW, Park SW, et al. 10th rib cartilage: another option of the costal cartilage graft for rhinoplasty. Arch Aesthetic Plast Surg 2015;21:47-53.

10. Park JH, Jin HR. Use of autologous costal cartilage in Asian rhinoplasty. Plast Reconstr Surg 2012;130:1338-48.

11. Frodel JL, Lee S. The use of high-density polyethylene implants in facial deformities. Arch Otolaryngol Head Neck Surg 1998;124:1219-23.

12. Han K, Jeong JW, Kim JH, et al. Complete septal extension grafts using porous high-density polyethylene sheets for the westernization of the Asian nose. Plast Reconstr Surg 2012; 130:106e-115e.

13. James SE, Kelly MH. Cartilage recycling in rhinoplasty: polydioxanone foil as an absorbable biomechanical scaffold. Plast Reconstr Surg 2008;122:254-60.

14. Boenisch M, Nolst Trenite GJ. Reconstructive septal surgery. Facial Plast Surg 2006;22:249-54.

15. Tweedie DJ, Lo S, Rowe-Jones JM. Reconstruction of the nasal septum using perforated and unperforated polydioxanone foil. Arch Facial Plast Surg 2010;12:106-13.

16. Kim JH, Song JW, Park SW, et al. Tip extension suture: a new tool tailored for Asian rhinoplasty. Plast Reconstr Surg 2014;134:907-16. 